\title{
IMPORTANCE OF NUCLEAR PHYSICS TO NASA'S SPACE MISSIONS
}

\author{
R.K. Tripathi ${ }^{1}$, J.W. Wilson ${ }^{1}$, and F.A. Cucinotta ${ }^{2}$ \\ (1) NASA Langley Research Center, Hampton, VA, USA; (2) Johnson Space Center, Houston, TX, USA
}

\begin{abstract}
We show that nuclear physics is extremely important for accurate risk assessments for space missions. Due to paucity of experimental input radiation interaction information it is imperative to develop reliable accurate models for the interaction of radiation with matter. State-of-the-art nuclear cross sections models have been developed at the NASA Langley Research center and are discussed.
\end{abstract}

\section{INTRODUCTION}

Accurate nuclear interaction databases are needed for describing the transport of space radiation in matter including spacecraft structures, atmospheres, and tissues. Transport models support the identification and development of new material concepts for human and electronic part protection. The success of the Human Exploration and Development of Space (HEDS) program in NASA critically depends on the minimizing the exposure to astronauts from Galactic Cosmic Rays in deep space for which there is, as yet, little human experience. The usual method for reduction of exposure is the design of shield for specific missions. The types and energy distributions of particles transmitted through a shield material require the solution to a transport description of the process with appropriate boundary conditions. The relevant transport equations are the linear Boltzmann equations derived on the basis of conservation principles for the flux density $\phi_{\mathrm{j}}(\mathbf{x}, \Omega, \mathrm{E})$ of type j particles at $\mathbf{x}$ of energy $\mathrm{E}$ moving in direction $\Omega$ as

$$
\Omega \bullet \nabla \phi_{j}(\mathbf{x}, \Omega, \mathrm{E})=\sum \int \sigma_{\mathrm{jk}}\left(\Omega, \Omega^{\prime}, \mathrm{E}, \mathrm{E}^{\prime}\right) \phi_{\mathrm{k}}\left(\mathbf{x}, \Omega^{\prime}, \mathrm{E}^{\prime}\right) \mathrm{d} \Omega^{\prime} \mathrm{dE} \mathrm{E}^{\prime}-\sigma_{\mathrm{j}}(\mathrm{E}) \phi_{\mathrm{j}}(\mathbf{x}, \Omega, \mathrm{E})
$$

where $\sigma_{\mathrm{j}}(\mathrm{E})$ and $\sigma_{\mathrm{jk}}\left(\Omega, \Omega^{\prime}, \mathrm{E}, \mathrm{E}^{\prime}\right)$ are the media macroscopic total and fragmentation cross sections. The $\sigma_{\mathrm{jk}}\left(\Omega, \Omega^{\prime}, \mathrm{E}, \mathrm{E}\right.$ ') represents all those processes by which type k particles moving in direction $\Omega$ ' with energy $\mathrm{E}^{\prime}$ produce a type $\mathrm{j}$ particle in direction $\Omega$ with energy E. Note that there may be several reactions that produce a particular product, and the appropriate cross sections for equation (1) are the inclusive ones. Accuracy of the input cross sections is of prime concern to the project. The design and thickness of shield sensitively depends on this input information significantly impacting on the payload and the feasibility of space missions.

\section{RADIATION INTERACTION MODELS}

Nuclear interaction cross section databases are required for the transport of cosmic rays with energies below $10 \mathrm{~A}$ $\mathrm{MeV}$ to energies above tens of $\mathrm{A} \mathrm{GeV}$, including a large number of projectile and target material combinations. The types of cross sections required for transport involve total yields and secondary energy spectra for one-dimensional transport and double differential cross sections in angle and energy for three-dimensional transport. Neutron and proton cross sections have been studied at some length in the past. Nuclear-reaction modeling is required, especially for both light and heavy ion projectiles, to understand the basic physical processes, and to extrapolate limited experimental data between projectile energies and projectile-target combinations. Our effort in developing nuclear cross sections models in NASA have largely focused in two directions (i) Semi-empirical / phenomenological and (ii). Microscopic. The emphasis in our work is on the accuracy of the results where experimental results are available and predicting reliable results where data are not available. The details of the models, comparisons with experiments and other challenging nuclear physics issues related to space missions will be discussed 


\section{Semi-empirical / Phenomenological Models}

The semi-empirical / Phenomenological model for fragmentation NUCFRG2 [1] and for absorption ABSXSEC [2], have modeled the essential physics. These models are coupled to the transport codes and, therefore, are meant to be very fast and accurate and give excellent results for the fragmentation and absorption cross sections.

\section{Fragmentation Cross Sections Model}

The semi-empirical fragmentation cross section model NUCFRG2 is an abrasion-ablation model and has been successful in generating the several thousand of energy dependent fragmentation cross sections for use in the study of high charge and energy (HZE) ion beams, radiation physics and galactic cosmic ray studies. The generated cross sections agree with the measured HZE fragmentation data to the degree that different experimental results agree among themselves. This version includes several improvements over NUCFRG1, including a Coulomb trajectory correction, an improved treatment of nuclear attenuation factors, an improved second order correction to the spectator fragment excitation spectrum, a pre-equilibrium emission process, and competitive equilibrium emission of additional hydrogen and helium isotope fragments. Fig. 1 shows the results obtained for charge removal, elemental cross sections and fluence for a few systems using NUCFRG2 model.
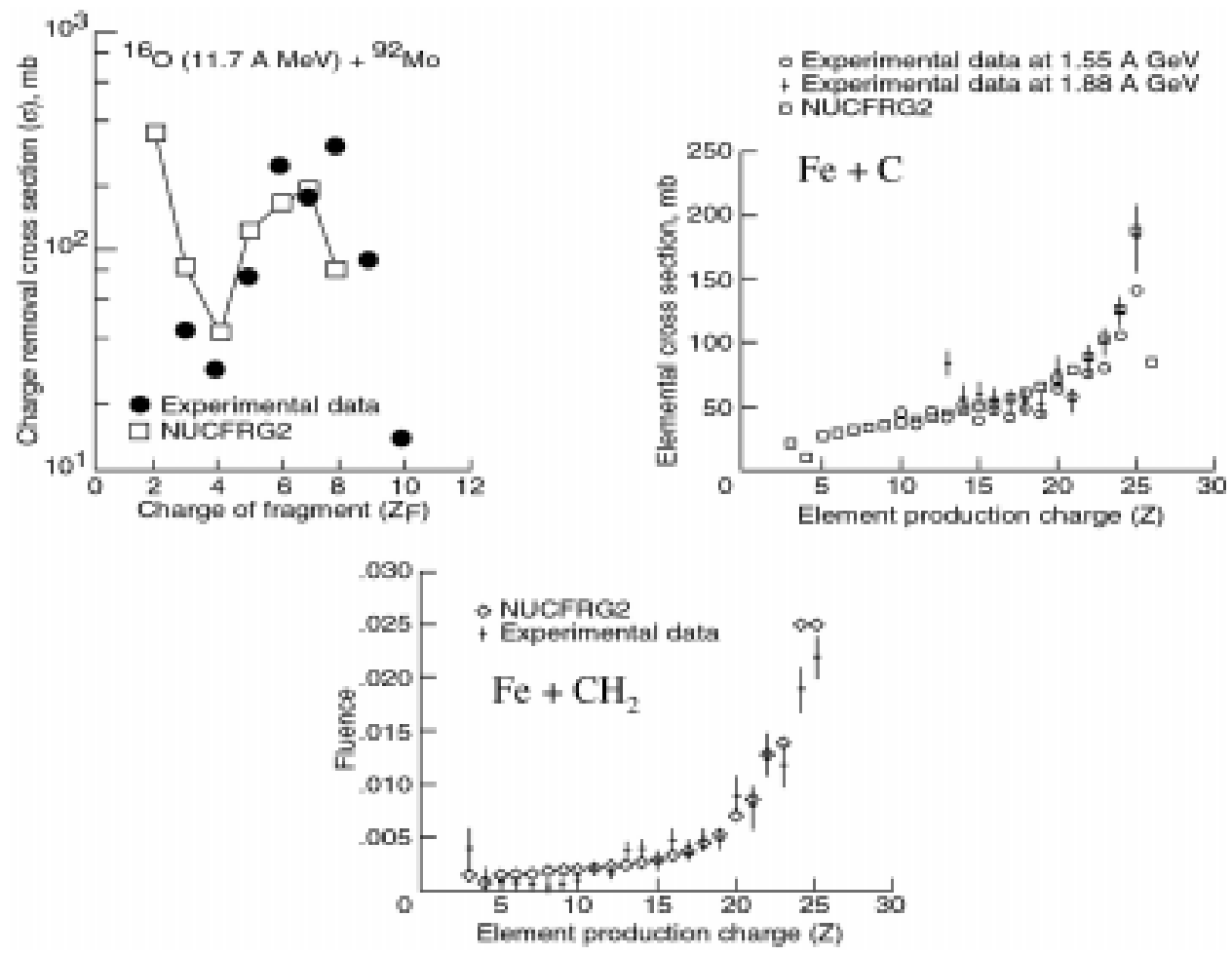

FIGURE 1. Charge removal, Elemental Cross Sections and Fluence for various systems using NUCFRG2 model.

\section{Absorption Cross Sections Model}

The ABSXSEC model calculates the absorption cross-sections for the collision of light, medium, heavy, charged and/or uncharged ions. The model gives excellent results for a wide rage of energies from a few $\mathrm{A} \mathrm{MeV}$ to a few $\mathrm{A}$ $\mathrm{GeV}$. ABSXSEC has become state-of-the art in the literature and is widely used here in the USA and else where around the world. Due to restrictions on export of software abroad, the code has been made available within USA. The model incorporates essentials aspects of the physics of the collision systems like Coulomb interaction, Pauli blocking, transparency, asymmetry and isotopic effects. Fig. 2 shows the results of the model for a few collision systems. It is gratifying to note the model has excellent agreement up to six orders of magnitude in energy (e.g. p + $\alpha)$. 

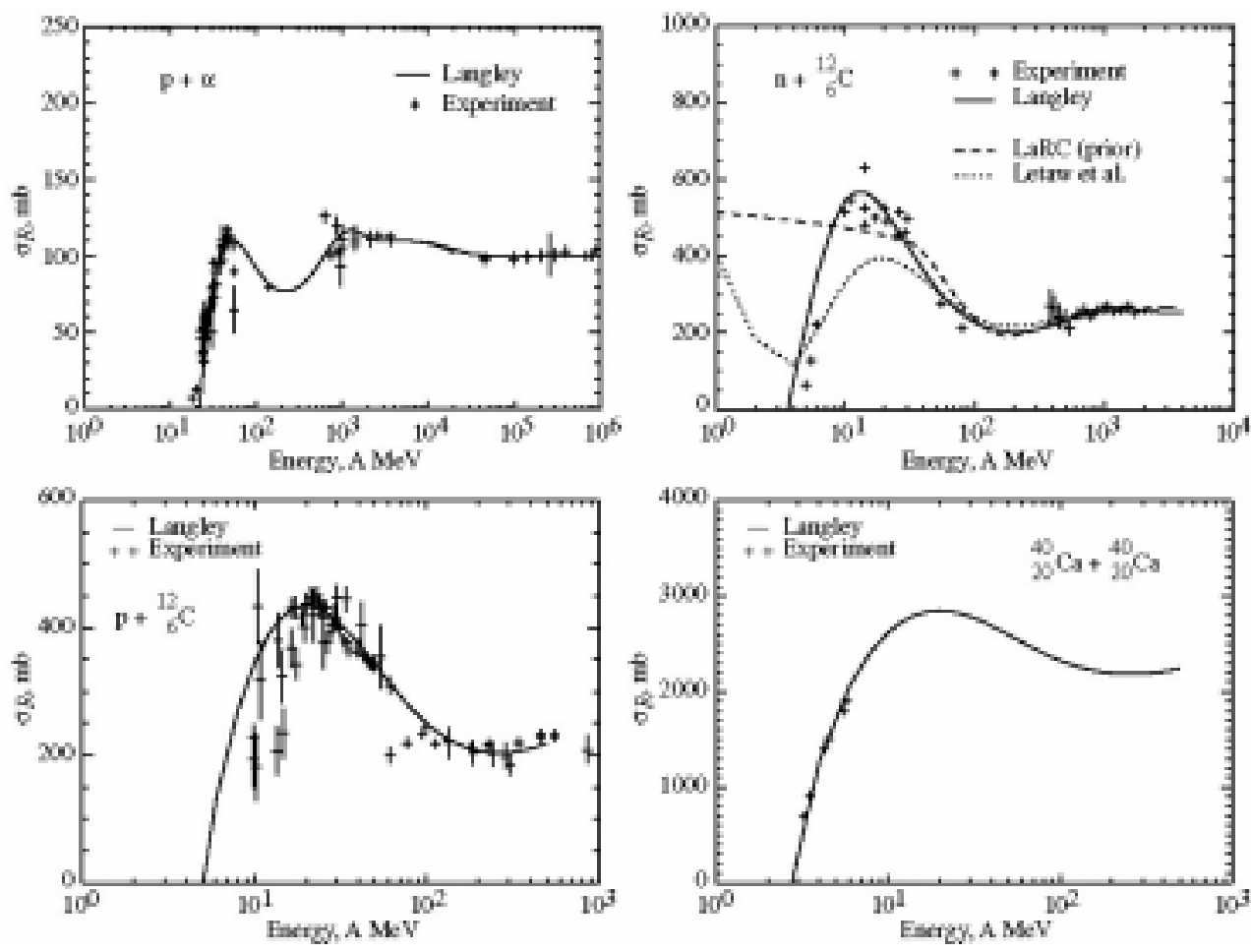

FIGURE 2. Absorption Cross Sections (ABSXSEC Langley Model)

\section{Microscopic Cross Sections Models}

The microscopic model for fragmentation QMSFRG [3] and the general purpose OPTICAL MODEL [4] are based on multiple scattering theory. For high-energy reactions, the eikonal approximation, which assumes that the scattering is peaked at the forward angles, simplifies the solution of multiple scattering series. OPTICAL MODEL, in addition, uses nucleon-nucleon (NN) cross sections and the ratio of the real to imaginary part of the two-body scattering amplitudes in the medium and calculates absorption, total and fragmentation cross sections thus providing a comprehensive picture of the input information for the radiation protection and shielding projects of NASA space missions. QMSFRG: incorporates statistical methods for the analysis of continuous excitation energy distribution of the pre-fragments. This approach allows nuclear structure and clustering effects to be considered. As a result, this model explains features like odd-even effects of the fragmentation cross sections. These models have been very successful in predicting experimental results.

\section{Quantum Multiple Scattering Model}

Quantum effects are manifested in nuclear reactions in several ways including interference effects between terms in the multiple scattering series, the many-body nuclear wave functions (for e.g. the roles of shell structure and Fermi momentum) and nuclear clustering. The quantum multiple scattering fragmentation model (QMSFRG) is a comprehensive model for generating nuclear interaction databases for galactic cosmic ray (GCR) transport. Quantum effects are especially important for heavy ions with mass numbers less than 20 that dominate radiation transport in human tissues and for the materials that are expected to be superior in space radiation protection. Our efforts in model developments have focused largely on quantum multiple scattering theories (QMST). In the QMST a the many-body integral-equation for the transition operator for nucleus-nucleus scattering [3] is considered,

$$
\mathrm{T}=\mathrm{K}+\mathrm{K} \mathrm{G} \mathrm{T}
$$

where $\mathrm{K}$ is the interaction kernel and $\mathrm{G}$ the Bethe-Salpeter propagator representing two nuclei in intermediate states. The kernel is the sum of all irreducible diagrams for projectile-target constituents. The solution of (2) is made using appropriate high energy approximations for momentum and energy transfers typical of nuclear reactions, 


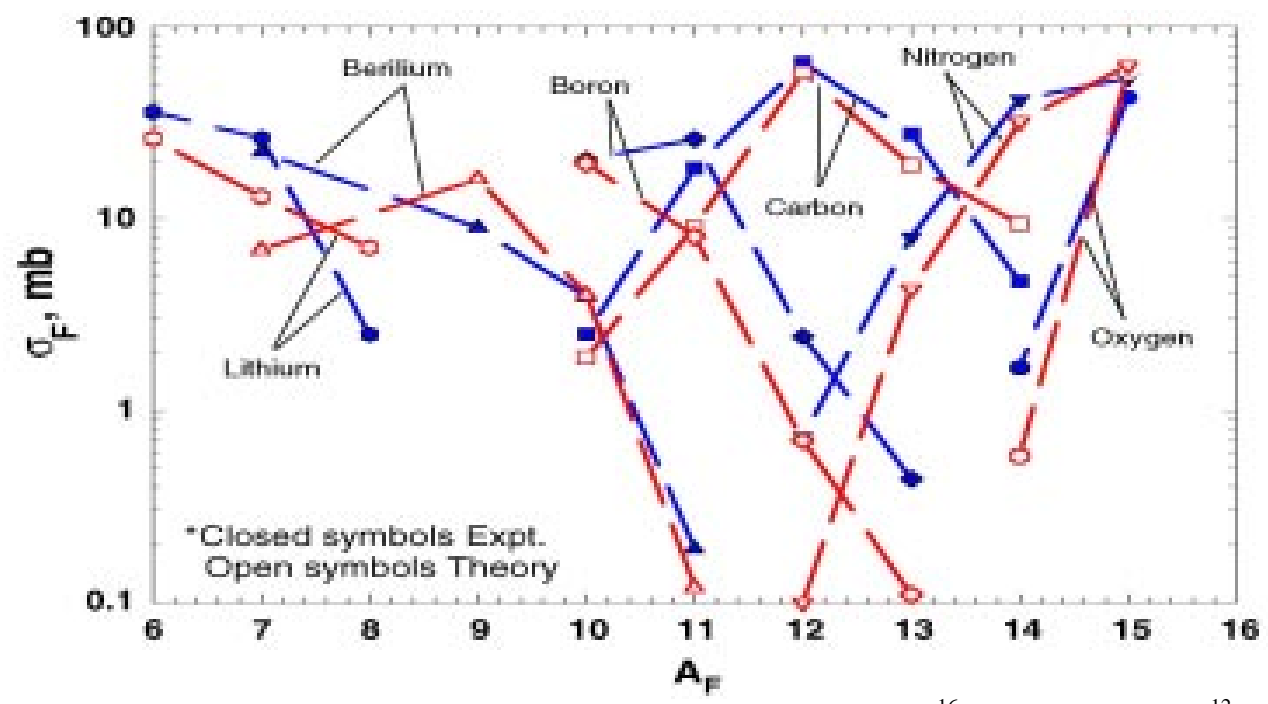

FIGURE 3. Comparison of QMSFRG to experiments for $2.1 \mathrm{~A} \mathrm{GeV}{ }^{16} \mathrm{O}$ fragmentation on ${ }^{12} \mathrm{C}$

development of bound state function models for transitions of interests, and new methods to deal with many particle phase problems for the description of fragmentation channels.

The production cross sections for the fragments are found by considering the phase space for an arbitrary final state where there are $\mathrm{n}$ abraded particles, leaving a projectile pre-fragment with energy denoted $\mathrm{E}_{\mathrm{F} *}$. Conserving energy in the pre-fragment formation after interactions with the target are complete, the scattering amplitude $\mathrm{f}_{\mathrm{fi}}$ and cross sections are related by

$$
\mathrm{d} \sigma=\Sigma_{\mathrm{X}} \Pi_{\mathrm{j}=1}^{\mathrm{n}}\left[\mathrm{dk}_{\mathrm{j}} /(2 \pi)^{3}\right] \delta\left(\mathrm{E}_{\mathrm{i}}-\mathrm{E}_{\mathrm{f}}\right) \mathrm{dE} \mathrm{E}_{\mathrm{F}} \mathrm{d}^{2} \mathrm{q}\left|\mathrm{f}_{\mathrm{fi}}\right|^{2}
$$

where the $\mathbf{k}_{\mathrm{j}}$ are the abraded nucleon wave vectors, $E_{\mathrm{i}}$ and $\mathrm{E}_{\mathrm{f}}$ are the energies in the initial and final states, and $q$ is the total transverse momentum in the reaction. Equation (3) is summed over the final states of the target nuclei, X. The neglect of the quantum interference terms in eq.(3) that the Monte-Carlo codes such as FLUKA and GEANT, based in classical physics, is a major source of inaccuracy in these models.

In the abrasion-ablation model there is a causal assumption that separates the time evolution of ablation processes from the abrasion. It follows that for the emission of $\mathrm{v}$ nuclei from the excited pre-fragment with energies $\mathrm{E}_{\mathrm{r}}$ we have $\mathrm{E}_{\mathrm{F}^{*}}=\mathrm{E}_{\mathrm{F}}+\Sigma_{\mathrm{r}}^{\mathrm{v}} \mathrm{E}_{\mathrm{r}}$ where $\mathrm{r}=0$ is allowed in order to include the possibility that the pre-fragment excitation energy is below the lowest particle-emission channel. The abraded particle momentum distribution is

$$
\mathrm{d} \sigma / \mathrm{dk}=\Sigma_{\mathrm{n}} \Sigma_{\mathrm{X}} \int \Pi_{\mathrm{j}=2}^{\mathrm{n}}\left[\mathrm{d} \mathbf{k}_{\mathrm{j}} /(2 \pi)^{3}\right] \mathrm{dE}_{\mathrm{F}^{*}} \mathrm{~d}^{2} \mathrm{q}\left|\mathrm{f}_{\mathrm{fi}}\right|^{2}
$$

where we are integrating over all variables except the momentum of one abraded particle. The excitation spectra of pre-fragment nuclei are obtained from equation (3) as

$$
\mathrm{d} \sigma / \mathrm{dE}_{\mathrm{F}^{*}}=\Sigma_{\mathrm{X}} \int \Pi_{\mathrm{j}=1}^{\mathrm{n}}\left[\mathrm{d} \mathbf{k}_{\mathrm{j}} /(2 \pi)^{3}\right] \mathrm{d}^{2} \mathrm{q}\left|\mathrm{f}_{\mathrm{fi}}\right|^{2}
$$

The decay of the pre-fragment nuclei into the final fragment opens the phase space further, and this description is required for predicting the final mass yields as well as momentum distributions of ablated nucleons or light nuclei. Ablation is described by solving the Master equation for de-excitation of the pre-fragments. For light nuclei experimental decay parameters are used. Fig. 3 shows results of the model for ${ }^{16} \mathrm{O}$ fragmentation and gives excellent agreement with experiment.

\section{Optical Model approach}

The model is based on coupled-channel multiple scattering approach (see reference 4 and references there in for details). In this the basic quantity is the matrix for elastic scattering amplitude, 


$$
f(\mathbf{q})=-\frac{i k}{2 \pi} \int d^{2} \mathbf{b} \exp (-i \mathbf{q} \cdot \mathbf{b})\{\exp [i \chi(\mathbf{b})]-1\}
$$

where $f$ and $\chi$ represent matrices, $\mathrm{k}$ is the projectile momentum relative to the center of mass, $\mathbf{b}$ is the projectile impact parameter vector, $\mathbf{q}$ is the momentum transfer, and $\chi(\mathbf{b})$ is the eikonal phase matrix. The total cross section can be easily obtained from the elastic scattering amplitude by using the optical theorem,

$$
\sigma_{\text {tot }}=4 \pi \int_{0}^{\infty} \mathrm{db} b\left\{1-\mathrm{e}^{-\operatorname{Im}(\chi)} \cos (\operatorname{Re}(\chi))\right\}
$$

And the absorption cross sections $\left(\sigma_{\mathrm{abs}}\right)$ is given by,

$$
\sigma_{a b s}=2 \pi \int_{0}^{\infty} d b b\left\{1-e^{-2 \operatorname{Im}(x)}\right\}
$$

Having calculated the total and absorption cross sections, for many nuclei, elastic cross section is easily obtained by the difference of these quantities,

$$
\sigma_{\mathrm{el}}=\sigma_{\mathrm{tot}}-\sigma_{\mathrm{abs}}
$$
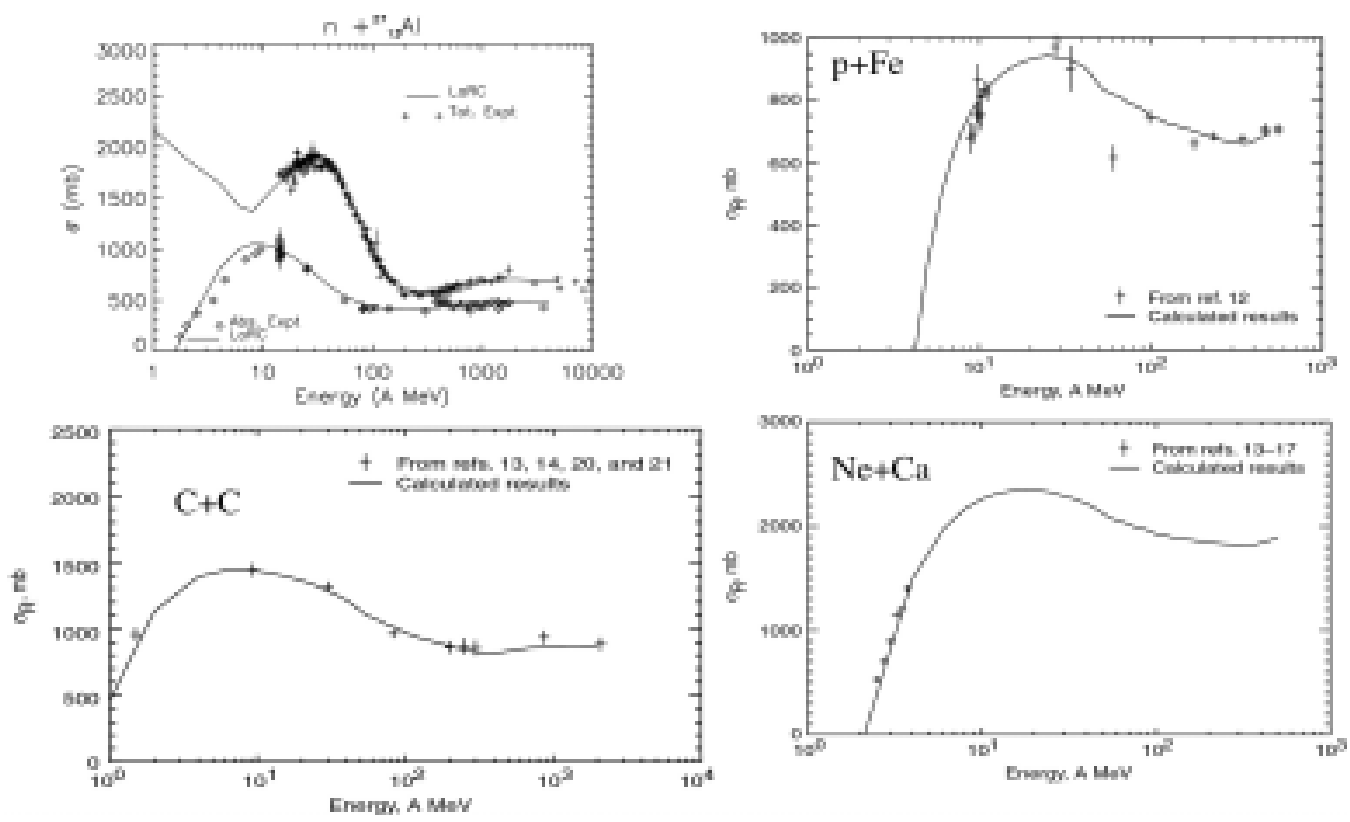

FIGURE 4. Absorption and Total Cross Sections (Optical Model)

Recently, we developed a method of extracting nucleon-nucleon $(\mathrm{N}-\mathrm{N})$ cross sections in the medium directly from experiment. The in-medium N-N cross sections form the basic ingredients of several heavy-ion scattering approaches including the coupled-channel approach developed at the NASA Langley Research Center. We investigated the ratio of real to imaginary part of the two body scattering amplitude in the medium. These ratios are used in combination with the in-medium N-N cross sections to calculate total, absorption and elastic proton-nucleus cross sections. The agreement with the available experimental data has been found to be excellent for all these cross section. Fig. 4 shows the examples of absorption and total cross sections for various systems.

\section{REFERENCES}

1. J.W. Wilson, R.K. Tripathi, F.A. Cucinotta, J.L. Shinn, F.F. Badavi, S.Y. Chun, J.W. Norbury, C.J. Zeitlein, L. Heilbronn and J. Miller, NASA TP 3533 (1995); Nucl. Instr. Meth. Phys. Res. B 94 (1994) 95

2. R.K. Tripathi, J.W. Wilson and F.A. Cucinotta, Nucl. Instr. and Meth. B 155 (1999) 349-356

3. F.A. Cucinotta, J.W. Wilson, J.L. Shinn and R.K. Tripathi, Adv. Space Res. 21 (12) (1998) 175

4. R.K. Tripathi, J.W. Wilson and F.A. Cucinotta, Nucl. Instr. And Meth. B 173 (2001) 391-396. 